\title{
Line Congruences of Low Order
}

\author{
Enrique Arrondo
}

\begin{abstract}
We present a survey on congruences of lines of low order. We start by recalling the main properties of the focal locus, and use it to reobtain the classification of congruences of order one in $\mathbb{P}^{3}$. We then explain the main ideas of a work in progress with S. Verra, outlining how to classify congruences of lines of order two in $\mathbb{P}^{3}$. We end by stating the main problems on this topic.
\end{abstract}

The study of line congruences (i.e. $(n-1)$-dimensional families of lines in $\mathbb{P}^{n}$ ), especially for $n=3,4$ has been very popular in the turn from nineteenth till twentieth century, and has been retaken with modern techniques at the end of the last century. One of the aspects studied has been the classification of congruences of low order (the order is defined to be the number of lines of the family passing through a general point of $\mathbb{P}^{n}$ ). In particular, line congruences in $\mathbb{P}^{3}$ of order up to three have been studied among many others by Kummer ([15]) and Fano ([9] and [10]), while congruences of order one in $\mathbb{P}^{4}$ have been studied especially by Marletta ([17] and [18]). Most of the results obtained by the classical geometers have been reobtained and improved in nowadays terms. Specifically, Ran ([20]) has given a complete classification of all the two-dimensional families of linear spaces of any dimension in any projective space and having order one (in particular, he reobtained, fixing one missing case, Kummer's classification of line congruences of order one in $\left.\mathbb{P}^{3}\right)$. The cases of order two and three (still in $\mathbb{P}^{3}$ ) were considered with modern techniques only when the family of lines was smooth. The classification of order two was obtained by Verra $([23])$, while the classification of order three was completed by Gross ([14]). For line congruences in $\mathbb{P}^{4}$, the situation is much more complicated, and in fact only partial classifications are known for order one, even in case 
the congruences are assumed to be smooth (see [7] and [8] for a thorough study of some of the cases outlined by Marletta).

In this paper we will give an overview of the general situation, explaining how to obtain much of the above classification, and enlarging it to the case of line congruences of order two in $\mathbb{P}^{3}$ without the smoothness hypothesis (the latter being a work in progress with Sandro Verra). The concrete structure and contents of the paper are as follows.

In Section 1 we will fix the notation and basic definitions, in particular the one of the focal locus, a classical topic recently retaken by Ciliberto and Sernesi ([5]). We will then state and briefly prove the main properties of the focal locus of line congruences that we will need. A complete study of these (and many other) properties in the case of line congruences in $\mathbb{P}^{3}$ (the main case treated in this paper) can be found in [3], while for an arbitrary $\mathbb{P}^{n}$ the results will appear in a forthcoming paper of Marina Bertolini, Cristina Turrini and the author. Anyway most of the results of this section (and the next one) are very classical and has been reproved in different ways by many authors.

In Section 2 we will explain the general situation for congruences of order zero or one. We will concentrate on line congruences of order one in $\mathbb{P}^{3}$, reobtaining the classification of Ran. I should mention that this very result has also been revisited by Zak, Inshakov, L'vovski and Oblomkov (see [24]), De Poi (see [6]), as well as Pedreira and Solá-Conde (see [19]).

In Section 3 we will see how to extend the techniques of the previous section to complete the classification of line congruences of order two in $\mathbb{P}^{3}$. Since this classification will appear in a joint paper with Sandro Verra, we will not give all the details. Instead we will prefer to clearly show how a few ideas will produce a classification in some particular cases. And rather than giving a precise description of each of these cases we will just show, for the cases admitting infinitely many degrees, that these cases are possible by just giving particular examples.

Finally in Section 4 we will briefly discuss some open problems about congruences of lines related to the topics covered in this paper.

ACKnowledgments. I would like to thank Cristina Turrini for helping me to improve the presentation of this paper. 


\section{General results and definitions}

We first fix the general notation that will be used in the paper.

Notation. Throughout the paper, $G(k, n)$ will denote the Grassmann variety of $k$-planes in the projective space $\mathbb{P}^{n}$. If $k=1, \Omega(A, B)$ (with $A \subset B \subset \mathbb{P}^{n}$ being linear subspaces) will denote the Schubert variety of lines meeting $A$ and contained in $B$. If $E$ is a locally free sheaf (which we will without warning identify with its corresponding vector bundle) over a projective variety $X, \mathbb{P}(E)$ will denote the projective bundle of rank-one quotients of $E$.

Definition 1.1. A line congruence will mean for us an irreducible subvariety $X$ of dimension $n-1$ in $G(1, n)$, the Grassmannian of lines in $\mathbb{P}^{n}$.

In other words, a line congruence consists of an $(n-1)$-dimensional family of lines in $\mathbb{P}^{n}$, so that one expects that the whole $\mathbb{P}^{n}$ will be covered by the lines of the congruence. This motivates the following definition.

Definition 1.2. The order of a line congruence is the number of lines that pass through a general point of $\mathbb{P}^{n}$.

Another invariant that we will use (especially when $n=3$ ) is the following.

Definition 1.3. The class of a line congruence is the number of lines contained in a general hyperplane $H \subset \mathbb{P}^{n}$ and meeting a general line $L$ of $H$. Observe that for $n=3$ the class is just the number of lines of the congruence contained in a general plane.

The simplest example consists of case $n=2$. A line congruence in $\mathbb{P}^{2}$ is nothing but a curve in the dual plane $\mathbb{P}^{2^{*}}$, and the order is the degree of such curve (the class of a congruence will always be zero in this case). In this situation it is well-known that a congruence (if it is not a pencil of lines, i.e. if the order is not one) is just the set of tangent lines to some curve in $\mathbb{P}^{2}$. In this paper we will be mainly interested in the first non-trivial case, i.e. when $n=3$.

The main construction associated with a line congruence is the following. First consider the incidence variety $I_{X} \subset X \times \mathbb{P}^{n}$ consisting of pairs $(L, p)$ for which the line $L$ contains the point $p$. We will consider the projection $q: I_{X} \rightarrow \mathbb{P}^{n}$ to the second factor. This map is generically finite (unless $X$ has order zero), and its degree is exactly the order of the congruence. We can then make the following definitions. 
Definition 1.4. The focal locus of a congruence is the branch locus $F \subset \mathbb{P}^{n}$ of the above map $q$ (hence it is expected to be a hypersurface in $\mathbb{P}^{n}$ ). A focus or focal point is just a point in the focal locus. If the pair $(L, p)$ is in the ramification locus of $q$, we will then say that $p$ is a focal point for the line L. Similarly, the fundamental locus of a congruence is the set of points in $\mathbb{P}^{n}$ for which the fiber of $q$ is infinite. Such a point is called fundamental point of the congruence. A fundamental curve of a congruence is a curve in $\mathbb{P}^{n}$ such that all its points are fundamental. Notice that if $n=3$ then any line of the congruence should meet the fundamental curve (assuming it exists), and in fact this property characterizes fundamental curves (except in the special case in which the congruence consists of the lines passing through a point).

In other words, a focus is a point through which there pass two infinitely close lines of the congruence, while a fundamental point is characterized by the fact that infinitely many lines of the congruence pass through it. Clearly, a fundamental point is always focal. Observe that on a congruence of order zero or one both definitions coincide. We state now a very well-known result, of which we will give a brief intuitive geometric proof (a complete rigorous proof in nowadays language can be found for instance in [12] or [3] for $n=3$; for arbitrary $n$ the proof works in the same way as in [3], and in fact the same authors are preparing a paper containing in particular such a proof).

LEMmA 1.1. Let $X$ be a line congruence in $\mathbb{P}^{n}$. Any line of $X$ either contains $n-1$ points (counted with multiplicity) that are focal for the line or all its points are focal. Moreover, the line is tangent to the focal locus at those points (provided they are smooth for the focal locus).

Proof. Given $L \in X$, we consider the restriction of $T_{I_{X}} \stackrel{d q}{\rightarrow} q^{*} T_{\mathbb{P}^{n}}$ to $L \cong \mathbb{P}^{1}$ (identified with the fiber of $L$ under the first projection $\pi: I_{X} \rightarrow X$ ). This morphism becomes then $\mathcal{O}_{\mathbb{P}^{1}}^{n-1} \oplus \mathcal{O}_{\mathbb{P}^{1}}(2) \rightarrow \mathcal{O}_{\mathbb{P}^{1}}(1)^{n-1} \oplus \mathcal{O}_{\mathbb{P}^{1}}(2)$ (use the exact sequence $0 \rightarrow T_{I_{X} / X} \rightarrow T_{I_{X}} \rightarrow \pi^{*} T_{X} \rightarrow 0$ restricted to $L$ ), whose degeneracy locus is thus given by a linear form of degree $n-1$ on $\mathbb{P}^{1}$. Hence this form either is identically zero or vanishes exactly at $n-1$ points counted with multiplicity.

We can give an alternative proof of this first fact, which will eventually help to prove the second fact in the statement. The fact that $p$ is a focal point for a line $L$ of a congruence $X$ means that the schematic intersection of $X$ with the set $\Omega\left(p, \mathbb{P}^{n}\right)$ (of lines passing through $p$ ) contains the line $L$ 
with multiplicity at least two. But a subscheme of length two contained in $\Omega\left(p, \mathbb{P}^{n}\right)$ is necessarily contained in a pencil $\Omega(p, \Pi)$ of lines contained in $\Pi$ and passing through $p$, for some plane $\Pi \subset \mathbb{P}^{n}$. Then $p$ is a focal point for $L$ if and only if there exists a pencil $\Omega(p, \Pi)$ meeting $X$ at $L$ with multiplicity at least two. In other words, the line in $G(1, n) \subset \mathbb{P}^{\frac{n(n+3)}{2}}$ represented by $\Omega(p, \Pi)$ is tangent to $X$ at $L$. Since any line of $\mathbb{P}^{\frac{n(n+3)}{2}}$ contained in $G(1, n)$ is necessarily a pencil of lines in $\mathbb{P}^{n}$, it follows that finding the focal points for $L$ is equivalent to finding the tangent directions of $X$ at $L$ that are contained in $G(1, n)$.

Now, regarding the line $L$ of the congruence as a point in $G(1, n) \subset$ $\mathbb{P}^{\frac{n(n+3)}{2}}$, the set of lines of $\mathbb{P}^{\frac{n(n+3)}{2}}$ passing through $L$ is a cone over the Segre embedding of $\mathbb{P}^{1} \times \mathbb{P}^{n-2}$ (where $\mathbb{P}^{1}$ is naturally identified with the set of points $p$ in the line $L$, while $\mathbb{P}^{n-2}$ is identified with the set of planes $\Pi$ of $\mathbb{P}^{n}$ containing $L$ ). Hence this is an $n$-dimensional cone (and it is exactly the intersection $\Omega\left(L, \mathbb{P}^{n}\right)$ of $G(1, n)$ with its embedded tangent space $T$ at the point $L)$ and it has degree $n-1$. Then the union of the lines $\Omega(p, \Pi)$ of $\mathbb{P}^{\frac{n(n+3)}{2}}$ we are looking for is exactly the intersection in $T$ (a projective space of dimension $2 n-2$ ) of this $n$-dimensional cone with the embedded tangent space of $X$ at $L$ (a projective space of dimension $n-1$ if $L$ is a smooth point of $X$ ). We thus obtain in general $n-1$ of such lines (counted with multiplicity).

Now proving the tangency condition becomes easier. Indeed let $p$ be a focal point for $L$ and let $\Pi$ be a plane such that $\Omega(p, \Pi)$ meets $X$ with multiplicity at least two at $L$. This means that in $\Omega(p, \Pi)$ there is an infinitesimally close line to $L$ in $X$. On the other hand, this infinitesimally close line will contain a focal point infinitesimally close to $p$ (at least if $p$ is a smooth point of the focal locus). But it is clear that the direction in which this infinitesimally close focal point approaches $p$ is necessarily the direction of $L$, so that $L$ is indeed tangent to the focal locus at $p$.

EXAMPLE 1.1. Lemma 1.1 applied to the case $n=2$ is just saying a very standard fact: that any curve in $G(1,2)=\mathbb{P}^{2^{*}}$ is nothing but the dual of a curve in $\mathbb{P}^{2}$ (this is not true when the curve consists of a pencil of lines; but then the focal locus is the center of the pencil, which should be considered to be singular, in the sense that its dimension is smaller than the expected one). But the lemma is also saying that the way of constructing such curve is just to take the focal locus of the original curve in $\mathbb{P}^{2^{*}}$. We also remark that it is very important to observe that Lemma 1.1 allows that, besides 
the $n-1$ focal points that we find at a general line, there could be other focal points, but these must be focal for lines different from the original one. Indeed if we take the set of lines tangent to a plane curve $C$ of degree $d$, any line $L$ of this congruence will contain not only the tangency point with $C$ (i.e. the point which is focal for $L$ ), but also $d-2$ other points (counted with multiplicity) of the focal locus $C$, but their tangent lines will be different to $L$, so these other points are not focal for $L$. And we can of course find bitangent lines to $C$, so with apparently two focal points of the congruence on it. But such lines will have multiplicity at least two (as points of the congruence), so that in fact all of their points are focal.

Definition 1.5. Given a line congruence $X \subset G(1, n)$ with order $a$ and class $b$, we will consider $C_{L}$ to be the curve consisting of the set of all lines of the congruence meeting a general fixed line $L$ (in particular, if $X$ is smooth in codimension one $C_{L}$ is smooth). In other words, $C_{L}=X \cap \Omega\left(L, \mathbb{P}^{n}\right)$. We will denote with $g_{X}$ the genus of $C_{L}$. Observe that $C_{L}$ is the congruence itself if $n=2$, while if $n=3, C_{L}$ is a hyperplane section of $X$ (after the Plücker embedding of $G(1,3)$ in $\left.\mathbb{P}^{5}\right)$. Since the hyperplane section of the Schubert class of $\Omega\left(L, \mathbb{P}^{n}\right)$ is the sum of the classes of $\Omega\left(p, \mathbb{P}^{n}\right)$ and $\Omega(L, H)$ (with $p$ a point of $\mathbb{P}^{n}$ and $H \subset \mathbb{P}^{n}$ a hyperplane containing $L$ ), it follows that the degree of $C_{L}$ is $a+b$.

With this definition, it is now easy to prove the following (again very well-known) result for the degree of the focal locus (observe that for $n=2$ it just gives the degree of the dual curve).

Lemma 1.2. The degree of the focal hypersurface (considered as a scheme) of a congruence is $2 a+2 g_{X}-2$, where $a$ is the order of the congruence.

Proof. This is just an immediate application of Hurwitz theorem to the natural map $C_{L} \rightarrow L$ (of degree $a$ ) associating to any element of $C_{L}$ (i.e. a line meeting $L$ ) its intersection point with $L$.

It is important to observe that the above degree is only valid as a scheme, since the focal locus can have non reduced components with some multiplicity (see [3] for some pathological examples). And we also point our attention that this is the degree as a hypersurface, i.e. it is zero in case the focal locus has smaller dimension. 


\section{Congruences of order zero and one}

\subsection{Order zero}

A congruence $X \subset \mathbb{P}^{n}$ has order zero if and only if the union of the lines of $X$ is not the whole $\mathbb{P}^{n}$. As we will see immediately, this means that the number of possibilities increases with $n$.

If $n=2$, it is clear that any one-dimensional family of lines in $\mathbb{P}^{2}$ must fill the whole $\mathbb{P}^{2}$, so there are no congruences of order zero (in fact this is just another way of stating the trivial fact that the degree of any curve in $\mathbb{P}^{2^{*}}$ is positive).

If $n=3$, the union of the lines of a congruence of order zero must be a surface $S \subset \mathbb{P}^{3}$. But it is very easy to check that an irreducible surface containing a two-dimensional family of lines is necessarily a plane (since any two points of $S$ can be then joined by a line contained in $S$ ). Therefore $X$ can only be the congruence of all the lines contained in a given plane.

If $n \geq 4$, the philosophy is the same as in case $n=3$, but things become more complicated. Now the union of the lines in $X$ is a variety $Y$ of dimension at most $n-1$. The point now is that for a variety of dimension $n-1$ the fact of containing an $(n-1)$-family of lines is very special, but not too much special if $n$ is big. As we have seen, for $n=3$ this would imply that $Y$ is a plane. But only for $n=4$ it is still possible to say something. We include the result in the following proposition.

Proposition 2.1. Let $X$ be a line congruence in $\mathbb{P}^{4}$. Then $X$ has order zero if and only if it is one of the following:

(i) The intersection in $G(1,4)$ of a hypersurface and some $G(1,3)$ (the latter interpreted as the set of lines contained in some hyperplane of $\left.\mathbb{P}^{4}\right)$.

(ii) The congruence of all the lines contained in a smooth quadric of $\mathbb{P}^{4}$ (hence $X$ is isomorphic to $\mathbb{P}^{3}$, and in fact its Plücker embedding in $\mathbb{P}^{9}$ coincides with the double Veronese embedding).

(iii) The congruence of all the lines contained in some plane of a onedimensional family of planes in $\mathbb{P}^{4}$.

Proof. It is immediate to check that the union of the lines of the congruence has dimension exactly three. Thus the only main ingredient is a theorem of B. Segre (see [22], or [21] for a modern proof) stating that a threefold with a three-dimensional family of lines is either a linear space of dimension three, or a three-dimensional quadric, or it contains a one-dimensional family of planes (and the given family of lines of the threefold consists of those contained in these planes). Clearly the last two cases correspond to cases 
(ii) and (iii) in the statement (we can assume the quadric to be smooth, since otherwise it would contain a one-dimensional family of planes and we reduce to case (iii)). In the first case, we have that the union of the lines of the congruence is a hyperplane in $\mathbb{P}^{4}$, so that $X$ is contained in some $G(1,3)$. But now we just use the very well-known fact that any hypersurface in $G(1,3)$ is the complete intersection of $G(1,3)$ with a hypersurface in $\mathbb{P}^{5}$. Extending this hypersurface to a hypersurface in $\mathbb{P}^{9}$ we see that we are in case (i).

We then see that even in this case in which we can give a classification, the possible cases are too many. Indeed we have infinitely many cases in (i) and (iii). For case (i) we can still say that essentially each possibility depends on the degree of the hypersurface. But cases in (iii) are parameterized by the choice of a curve in $G(2,4)$ (i.e. of the one-dimensional family of planes). Only if we impose the condition that $X$ is smooth we get a finite number of possibilities in (iii) (see [2]), but clearly we still have infinitely many cases in (i).

\subsection{Order one}

The complexity of congruences of order zero can give an idea of how complicated it can become for order one or two if $n \geq 4$. This is why we will restrict ourselves essentially to case $n=3$. In fact, already for $n=4$, even a classification of smooth congruences of order one is not known. The main result (again very well-known) to be used for congruences of order one is the following.

Lemma 2.1. Let $X$ be a line congruence of order one. Then its focal locus coincides with its fundamental locus, which has dimension at most $n-2$ and any line of the congruence meets it.

Proof. Through any focal point there pass two infinitely close lines of the congruence, which implies (since the order is one) that in fact there pass infinitely many lines of the congruence. Hence the focal locus coincides with the fundamental one. We also know from Lemma 1.1 that any line of the congruence meets the focal locus $F$. It it were $\operatorname{dim} F=n-1$, since through any point of $F$ there passes at least a one-dimensional family of lines of $X$ (which has dimension $n-1$ ) this would mean that any line of $X$ contains a one-dimensional family of points of $F$, i.e. any line of $X$ would be contained in $F$. But this would imply that $X$ has order zero, which is a contradiction. 
EXAMPLE 2.1. Just to give the flavor of how this focal method works, let us apply the previous result to the case $n=2$. In this case, Lemma 2.1 says that, given a line congruence $X$ in $\mathbb{P}^{2}$ of order one, there is a point through which all the lines of the congruence pass. This implies, comparing dimensions, that $X$ is the congruence of all the lines passing through that point. We thus recover the standard result that any curve in $\mathbb{P}^{2 *}$ of degree one is a pencil of lines.

We devote now the rest of this section to outline the classification of line congruences in $\mathbb{P}^{3}$ of order one (the precise statement is given in Theorem 2.1 below). So let $X \subset G(1,3)$ be such a congruence, and let $F$ be its focal locus. If $\operatorname{dim} F=0$, then as in the above example, $X$ should consist of the congruence of all the lines passing through one point.

We therefore assume from now on that $F$ is a curve. Since any line of the congruence contains two (maybe infinitely close) points that are focal for the line, it could happen that this fundamental locus $F$ has one or two components. Let us study these two cases separately.

CASE 1 . If $F$ has two components $F_{1}$ and $F_{2}$, this means that any line of $X$ meets both $F_{1}$ and $F_{2}$. Let $d_{1}:=\operatorname{deg} F_{1}$ and $d_{2}:=\operatorname{deg} F_{2}$. The set of lines meeting both $F_{1}$ and $F_{2}$ has clearly order (and also class) $d_{1} d_{2}$ (fixing a point $p \in \mathbb{P}^{3}$ the lines of $X$ passing through $p$ are those in the intersection of the cones over $F_{1}$ and $F_{2}$ with vertex $p$ ). The only way in which it can contain a congruence of degree one is that $F_{1}$ and $F_{2}$ meet in $d_{1} d_{2}-1$ points (counted with multiplicity). But these are too many points, as the following easy lemma shows.

Lemma 2.2. Let $F_{1}, F_{2}$ be two curves in $\mathbb{P}^{3}$ of respective degrees $d_{1}$ and $d_{2}$. If the intersection of $F_{1}$ and $F_{2}$ is a scheme of length $d_{1} d_{2}-1$, then $F_{1}$ and $F_{2}$ are rational, and one of them is a line.

Proof. For a general point $p \in F_{1}$ we can consider the cone $C_{p}$ over $F_{1}$ with vertex $p$. If $F_{1}$ is not a line, then $C_{p}$ is a surface of degree $d_{1}-1$, which meets $F_{2}$ in at least $d_{1} d_{2}-1$ points (counted with multiplicity). Then Bézout's Theorem implies that $d_{1} d_{2}-1 \leq\left(d_{1}-1\right) d_{2}$, which implies that $d_{2} \leq 1$. i.e. $F_{2}$ is a line. And it is clear that a curve of degree $d_{1}$ meeting a line in $d_{1}-1$ points is necessarily rational.

Therefore we conclude that in this case the congruence consists of the closure of the set of lines spanned by a point in a line $L$ and a different 
point in a rational curve $C$ of some degree $d$, with $C$ and $L$ meeting in a scheme of length $d-1$. This is a congruence of class $d$.

CASE 2. If $F$ is irreducible, this apparently implies that any line of the congruence is bisecant to $F$ (at the two focal points of the line). Therefore $X$ is in this case the congruence of all the bisecants to $F$. But now it is a well-known fact that an irreducible nondegenerate curve in $\mathbb{P}^{3}$ with only one apparent node is necessarily a twisted cubic. Hence $X$ is the congruence of all the bisecants to a twisted cubic, and in particular it is a congruence of class three.

However we are missing one possibility (this is why we used before the word "apparently"). In fact it could happen a priori that the two focal points on a line are always infinitely close. Let us analyze closer this possibility.

As we have seen in the proof of Lemma 1.1, the two focal points on a general line $L \in X$ came from the two tangent directions at $L$ that are contained in $G(1,3)$. These two tangent directions come from the intersection in $\mathbb{P}^{5}$ of $G(1,3)$ and the embedded tangent plane of $X$ at $L$. It is well-known that the embedded tangent plane is contained in $G(1,3)$ for any line if and only if $X$ is either the congruence of all the lines passing through a fixed point or the congruence of all the lines contained in a fixed plane (see for instance [12], Corollary 4.5.1). So that we can assume that the intersection of $G(1,3)$ with the embedded tangent plane at a general $L \in X$ is always a degenerate conic. If this conic consists of a couple of lines, we then actually get two tangent directions at a general $L$ that are contained in $G(1,3)$. They should correspond to two pencils of lines $\Omega\left(p_{1}, \Pi_{1}\right)$ and $\Omega\left(p_{2}, \Pi_{2}\right)$. The fact that both focal points coincide means that $p_{1}=p_{2}$. But then the plane in $\mathbb{P}^{5}$ generated by the lines $\Omega\left(p_{1}, \Pi_{1}\right)$ and $\Omega\left(p_{2}, \Pi_{2}\right)$ is contained in $G(1,3)$ (it is in fact the plane $\Omega\left(p_{1}, \mathbb{P}^{3}\right)=\Omega\left(p_{2}, \mathbb{P}^{3}\right)$ ), contrary to our assumption. As a consequence, the intersection of $G(1,3)$ with the embedded tangent plane at a general $L \in X$ must be a double line.

Let us prove now that $F$ is a line. Assume for contradiction that $F$ is not a line. Take then a plane $\Pi \subset \mathbb{P}^{3}$ meeting $F$ transversally in at least two different points $p_{1}$ and $p_{2}$. Since through each $p_{i}$ there pass infinitely many lines of $X$, at least one of them, say $L_{i}$, is contained in $\Pi$. But then $L_{1}$ and $L_{2}$ meet at one point $p$, which therefore is also focal, so that we have a line, for instance $L_{1}$, containing two different focal points, and therefore infinitely many (if $L_{1}=L_{2}$, then the same reasoning would show that this 
line is entirely focal). Hence $F=L_{1}$ and we have that the focal locus is contained in $\Pi$, contrary to the choice of $\Pi$ (also the fact that $F$ is a line contradicts our hypothesis).

We have thus proved that the focal locus $F$ is a line. The same argument as above shows that, for any plane containing $F$, the set of lines contained in it (there are necessarily infinitely many, since $F$ is fundamental) should consist of a pencil of lines with center at a point in $F$. We thus have a map from $F^{*}$ (the pencil of planes containing $F$ ) to $F$ that associates to each plane the center of the pencil of lines just described. For any such a finite map (of degree say $d$ ) we clearly have a congruence of order one (and class $d$ ), so that this finishes the classification.

We summarize here the classification we have got.

TheOREM 2.1. The complete list of congruences of order one in $\mathbb{P}^{3}$ is the following:

(i) The set of all the lines passing through a fixed point of $\mathbb{P}^{3}$.

(ii) The set of all the bisecants to a twisted cubic.

(iii) The closure of the set of lines joining a point of a rational curve $C \subset \mathbb{P}^{3}$ of degree $d$ and a different point of a line $L$ which is $(d-1)$-secant to $C$.

(iv) Given a line $L \subset \mathbb{P}^{3}$ and finite map $f: L^{*} \rightarrow L$ (where $L^{*}$ is the the pencil of planes containing $L)$, the union of the pencils $\Omega(f(\Pi), \Pi)$ when $\Pi$ varies in $L^{*}$.

REMARK 2.1. Note that case (iv) in Theorem 2.1 can be considered as a degeneration of case (iii), in which the rational curve $C$ of degree $d$ degenerates into the line $L$ (endowed with a multiple structure). Indeed, in this general case we have an isomorphism from $L^{*}$ (the pencil of planes containing $L$ ) to $C$. And the set of lines of the congruence in each plane of $L^{*}$ is exactly the pencil of lines through its image by the above isomorphism. Hence when $C$ degenerates to become a scheme of degree $d$ over $L$ we get the construction in case (iv). This degenerate case was missing in Kummer's classification and it was Ran to fix it.

REMARK 2.2. If we are interested in finding the smooth congruences of order one in $\mathbb{P}^{3}$, we observe that cases (i) and (ii) in Theorem 2.1 are smooth (they are in fact respectively a plane and the Veronese surface inside $\mathbb{P}^{5}$ ). Taking into account the above Remark 2.1, we observe that smooth congruences of type (iv) can only be found if we find smooth congruences of type (iii) for the same degree $d$ (since a degeneration of a singular congruence cannot be smooth). But if we consider the congruence of all the lines 
meeting both a rational curve $D$ of degree $d$ and a line $L$ that is $(d-1)$ secant to $D$, it is easy to see that the line $L$ belongs to the congruence with multiplicity $d-1$. Hence a congruence of type (iii) can be smooth only when $d=1,2$. And in fact it is not difficult to see that for both degrees we obtain smooth congruences.

\section{Congruences of order two}

In case of order two, we cannot say that any focal point is fundamental, but we are still in the limit situation in which it is possible to say something about the relation among the focal locus and the fundamental locus. The main observation is the following.

Lemma 3.1. Let $X$ be a line congruence in $\mathbb{P}^{n}$ of order two. If the focal locus of $X$ has degree bigger than $2 n-2$, then $X$ has a fundamental locus which is met by any line of the congruence.

Proof. Take a general line $L$ of the congruence. Since it is tangent at $n-1$ points of the focal locus, the fact that the degree of this locus is bigger than $2 n-2$ implies that $L$ meets $F$ at another point $p$ that is not focal for $L$ (of course one should also check degenerate cases, for instance when some of the $n-1$ focal points coincide for a general line, but we omit this kind of details). This means that $p$ is focal for another line $L^{\prime} \in X$. But then through $p$ there pass at least three lines of the congruence $\left(L^{\prime}\right.$ counted twice and $L$ ). Therefore $p$ is a fundamental point, and any line of $X$ meets the fundamental locus.

EXAMPLE 3.1. As we did for order one, we observe that the previous lemma gives a know result in the case $n=2$. In fact, since an irreducible curve in $G(1,2)$ of order two cannot have fundamental points (otherwise it would contain a component consisting of a pencil of lines through a point), Lemma 3.1 implies that the focal locus should have degree two (clearly degree one is impossible). We therefore obtain the standard result that any irreducible curve of degree two in $\mathbb{P}^{2 *}$ is the set of tangent lines to a conic.

From now on we will concentrate only on line congruences in $\mathbb{P}^{3}$ of order two. Thus what follows has been made in collaboration with Sandro Verra, and it will be published (with all the details and hopefully with a complete classification) elsewhere. Our main scope in this section will be to show that, except for a finite number of cases, a line congruence of order 
two in $\mathbb{P}^{3}$ must be of a very particular type (precisely as in Remarks 3.1 and 3.2).

A first remark is that Lemma 3.1 should reasonably imply from Lemma 1.2 that the sectional genus $g_{X}$ of a congruence of order two without a fundamental curve is one. But this would be significant only if we know that the congruence is not too singular. We in fact have the following.

Lemma 3.2. Let $X$ be a line congruence in $\mathbb{P}^{3}$ of order two. If it has no fundamental curve, then $X$ has at most isolated singularities. Moreover they should have multiplicity two.

Proof. Assume $X$ has a curve of singular points. Then any line $L$ of the congruence would meet another line $L^{\prime}$ that is singular for $X$. But then through the intersection point of $L$ and $L^{\prime}$ there would pass at least three lines ( $L^{\prime}$ counted twice and $L$ ). Hence this is a fundamental point of the congruence. Clearly we would obtain a curve of fundamental points when varying $L$ in $X$. A similar reasoning shows that, if $X$ possesses a line of multiplicity at least three, then any point on it would be fundamental, and hence such a line should be a fundamental curve.

We can now prove a first result about congruences of order two.

Proposition 3.1. Let $X$ be a line congruence in $\mathbb{P}^{3}$ of order two. If $X$ has no fundamental curve, then its class is at most 28 , or equivalently its degree is at most 30.

Proof. Since all the lines of the congruence are bitangent to the focal surface, which by Lemma 3.1 has degree at most four, it follows that the class $b$ of the congruence is bounded by the number of bitangents to a plane quartic. Therefore $b \leq 28$ and the degree of $X$ as a surface in $\mathbb{P}^{5}$ is at most 30 .

We should remark that this result is not sharp at all. In fact, as we observed before Lemma 3.2, one should expect to obtain that $g_{X}$ (which is now the sectional genus of $X$ as a surface in $\mathbb{P}^{5}$ ), is one, so that for instance from [16] Theorem 3 one should get that the degree of $X$ is at most nine.

After Proposition 3.1, we can assume from now on that $X$ possesses a fundamental curve $C \subset \mathbb{P}^{3}$ (by considering if necessary one of its components we can assume $C$ to be irreducible). A priori all the lines of $C$ could be bisecant to $C$. We will assume this is not the case, since it is not difficult to see (comparing Castelnuovo's inequality and the genus formula for a plane curve) that a curve $C$ has two apparent nodes (i.e. the congruence 
of bisecants to $C$ has order two) if and only if $C$ is a smooth elliptic quartic curve or a rational quartic with a double point.

Let us see now that the fact that $X$ has order two imposes strong restrictions on the fundamental curve $C$.

Proposition 3.2. Let $C \subset \mathbb{P}^{3}$ be an irreducible fundamental curve of a line congruence $X$ in $\mathbb{P}^{3}$ of order two. Assume $C$ is not rational. Then $C$ is hyperelliptic and there is a line $L \subset \mathbb{P}^{3}$ such that the hyperelliptic structure of $C$ is given by a map of degree two from $C$ to the pencil $L^{*}$ of planes containing $L$ so that each point is contained in the plane given by its image. Moreover, the lines of the congruence are given by the lines in the pencils defined by a point of $C$ and its corresponding plane in $L^{*}$.

Proof. Let $e$ be the degree of the cone formed by the lines of the congruence passing through a general point of $C$. Consider the projective space $\mathbb{P}^{N}$ (with $N+1=\left(\begin{array}{c}e+3 \\ 3\end{array}\right)$ ) consisting of all the surfaces of degree $e$ in $\mathbb{P}^{3}$. We therefore have a map $f: C \rightarrow \mathbb{P}^{N}$ associating to each point of $C$ its corresponding cone (to be rigorous, we should rather use a normalization of $C$, but we abuse notation to make the exposition clearer). The fact that $X$ has order two (together with the fact that a general line of the congruence meets $C$ in just one point) implies that the product of the degree of $f$ and the degree of its image is two. We have thus two possibilities:

(i) The degree of the map $f$ is one and its image has degree two. This means that $C$ is birational to its image and that its image is an irreducible conic, hence rational, contrary to our hypothesis.

(ii) The degree of the map is two and its image is a line. But this means in particular that each cone has at least two vertices (the two points of $C$ in its fiber by $f$ ). This implies that the cone contains at least one plane, and the pencils of lines defined by the plane and the two vertices on $C$ are contained in the congruence. We have then a map of degree two $f^{\prime}: C \rightarrow$ $\mathbb{P}^{3^{*}}$ associating to each point of $C$ its corresponding plane. The fact that the order of the congruence is two implies that the image of $f^{\prime}$ is a pencil of planes, so that we get the description in the statement.

EXAMPLE 3.2. Even if it is not the most general case, let us see that, for any degree of $C$, we can have the hyperelliptic case described in Proposition 3.2. To this aim, take $C$ to be a curve of bidegree $(2, d-2)$ in a smooth quadric of $\mathbb{P}^{3}$ and let $L$ be a $(d-2)$-secant line to $C$ (which can be certainly found inside the quadric). Thus $C$ is a hyperelliptic curve of degree $d$, and we can define $f$ to assign to each $p \in C$ the plane spanned by $p$ and $L$. In 
other words, we get the congruence consisting of the closure of the set of lines meeting one point of $C$ with another (distinct) point of $L$.

We will concentrate now, after Proposition 3.2, in the case in which there is a rational fundamental curve $C$. We will also assume that the congruence has no fundamental line and that it does not consist of the lines of a one-dimensional family of pencils (these two particular cases will be analyzed at the end of the section, in Remarks 3.1 and 3.2). We consider then the normalization $\varphi: \mathbb{P}^{1} \rightarrow C \subset \mathbb{P}^{3}$ of the fundamental curve $C$ and the vector bundle $E:=\varphi^{*}\left(\Omega_{\mathbb{P}^{3}}(2)\right)$. The projective bundle $\mathbb{P}(E)$ gives thus a desingularization of the subset of $G(1,3)$ consisting of all the lines meeting $C$. Hence there is a surface $\tilde{X} \subset \mathbb{P}(E)$ that is birational to $X$ under the natural map $\pi: \mathbb{P}(E) \rightarrow G(1,3)$. On the other hand, our choice of the twist in $E$ implies that the class $t$ of the tautological line bundle $\mathcal{O}_{\mathbb{P}(E)}(1)$ of $\mathbb{P}(E)$ is the pull-back by $\pi$ of the hyperplane section of $G(1,3)$. Using that $E$ is a vector bundle on $\mathbb{P}^{1}$ we can write

$$
E \cong \mathcal{O}_{\mathbb{P}^{1}}\left(a_{1}\right) \oplus \mathcal{O}_{\mathbb{P}^{1}}\left(a_{2}\right) \oplus \mathcal{O}_{\mathbb{P}^{1}}\left(a_{3}\right)
$$

with $a_{1}, a_{2}, a_{3} \in \mathbb{Z}$. Our final goal is to see that only finitely many values of $a_{1}, a_{2}, a_{3}$ are possible under our assumptions. We start by proving the following.

Proposition 3.3. If $C$ is not a plane curve, then $C$ is a twisted cubic, the set of lines of the congruences through a general point of $C$ consists of a quadratic cone and the class of the congruence is six.

Proof. Let $d$ be the degree of $C$. Thus $\varphi^{*} \mathcal{O}_{C}(1) \cong \mathcal{O}_{\mathbb{P}^{1}}(d)$ (to avoid confusion about the meaning of a twist, we will always specify with the corresponding subindex if we are working on $C$ or $\mathbb{P}^{1}$ ). Hence, we have that

$$
a_{1}+a_{2}+a_{3}=c_{1}(E)=c_{1}\left(\varphi^{*}\left(\Omega_{\mathbb{P}^{3}}(2)\right)\right)=2 d .
$$

On the other hand, we can write the class of $\tilde{X}$ inside $\mathbb{P}(E)$ as

$$
[\tilde{X}]=e t-D
$$

where $D$ is the pull-back of a divisor on $\mathbb{P}^{1}$, and $e$ is the degree of the cone formed by the lines of $X$ through a general point of $C$. We will abuse notation and freely identify $D$ with the divisor on $\mathbb{P}^{1}$ from which it comes and with its degree. It is not difficult to see that the degree of $X$ in $\mathbb{P}^{5}$ is $\int_{\mathbb{P}(E)}[\tilde{X}] \cdot t^{2}=2 e d-D$. Also the class $b$ of $X$ is $e d$ (given a general plane $\Pi \subset \mathbb{P}^{3}$, it meets $C$ in $d$ points, and at each of these points the corresponding 
cone has exactly $e$ lines contained in $\Pi$ ). Therefore, the order of $X$ is given by $e d-D$. We thus get that

$$
D=e d-2 .
$$

Pulling back to $\mathbb{P}^{1}$ the Euler sequence on $\mathbb{P}^{3}$ we get the exact sequence

$$
0 \rightarrow E(-d) \rightarrow H^{0}\left(\mathbb{P}^{3}, \mathcal{O}_{\mathbb{P}^{3}}(1)\right) \otimes \mathcal{O}_{\mathbb{P}^{1}} \rightarrow \mathcal{O}_{\mathbb{P}^{1}}(d) \rightarrow 0 .
$$

Since $C$ is not a plane curve, we must have $H^{0}\left(\mathbb{P}^{1}, E(-d)\right)=0$, so that

$$
a_{1} \leq a_{2} \leq a_{3} \leq d-1 .
$$

We observe now that $\tilde{X}$ is the zero locus of a nonzero element $s \in H^{0}\left(\mathbb{P}(E), \mathcal{O}_{\mathbb{P}(E)}(e t-D)\right) \cong H^{0}\left(\mathbb{P}^{1},\left(S_{y m m}^{e} E\right)(-D)\right)$. But the direct summand of $\left(S_{y m m}^{e} E\right)(-D)$ of maximum degree is $\mathcal{O}_{\mathbb{P} 1}\left(e a_{3}-D\right)$, while $e a_{3}-D=e a_{2}-e d+2 \leq e(d-1)-e d+2=2-e$. Hence necessarily $e \leq 2$, so that $e=2$ since we are assuming that the congruence is not made out of pencils.

We thus have $\left(\right.$ Symm $\left.^{e} E\right)(-D) \cong \mathcal{O}_{\mathbb{P}^{1}}\left(2 a_{1}-D\right) \oplus \mathcal{O}_{\mathbb{P}^{1}}\left(a_{1}+a_{2}-D\right) \oplus$ $\mathcal{O}_{\mathbb{P}^{1}}\left(a_{1}+a_{3}-D\right) \oplus \mathcal{O}_{\mathbb{P}^{1}}\left(2 a_{2}-D\right) \oplus \mathcal{O}_{\mathbb{P}^{1}}\left(a_{2}+a_{3}-D\right) \oplus \mathcal{O}_{\mathbb{P}^{1}}\left(2 a_{3}-D\right)$. This splitting can be interpreted as giving the coefficients of a quadratic form on the plane parameterizing the set of lines through a point of $C$ (a choice of coordinates for this plane has been given by the splitting of $E$ ). This quadratic form should not define a double line for a general point of $C$, and hence $0 \leq a_{2}+a_{3}-D=a_{2}+a_{3}-2 d+2$. Therefore $a_{2}=a_{3}=d-1$ and thus $a_{1}=2$. Since the quadratic form does not split (otherwise $X$ will consists of a one-dimensional family of pencils), then it should depend on the first coordinate (the one corresponding to $\mathcal{O}_{\mathbb{P}}\left(a_{1}\right)$ ). So we also have $0 \leq a_{1}+a_{3}-D=a_{1}+a_{3}-2 d+2$, which implies $a_{1}=d-1$ and therefore $d=3$. Hence $C$ is a twisted cubic and the class of the congruence is $e d=6$.

But it is now clear that the last part of the previous proof should work also for a plane curve. We in fact have the following.

Proposition 3.4. If the fundamental curve $C$ is plane (but not a line, as we are assuming), then $C$ has degree at most four and the class of the congruence is at most twelve.

Proof. Keeping the notations of the proof of Proposition 3.3, we have now that $a_{3}=d$ and $d-a_{2}=a_{1} \leq a_{2} \leq d-1\left(a_{2}=d\right.$ would imply that $C$ is a line). Since the form of degree $e$ on the plane a general point of $C$ does 
not contain a double line, it still follows (since we are assuming $e>1$ ) that at least $0 \leq(e-1) a_{2}+a_{3}-D=(e-1)\left(a_{2}-d\right)+2$, so that either $a_{2}=d-2$ and $e=2$ or $a_{2}=d-1$ and $e \leq 3$. But again we want that the general homogeneous form does not contain a linear form. Since $e \leq 3$ this is equivalent to impose that the general form does not split. We thus need at least $0 \leq a_{1}+(e-1) a_{3}-D=a_{1}-d+2$. This implies that $a_{1}$ (which is $d-a_{2}$, hence at most 2) is at least $d-2$. Therefore $d \leq 4$ and thus $X$ has class at most 8 if $e=2$, or 12 if $e=3$.

We end this section with a brief analysis of the two cases we did not consider yet. Observe that, together with the case in Proposition 3.2 (which is in fact a particular case of both cases) they are the only cases admitting arbitrarily high class.

REMARK 3.1. If a line congruence $X$ of order two has a fundamental line $C$, then any plane in $C^{*}$ (the pencil of planes containing $C$ ) contains infinitely many lines of $X$. Since the order is two, there are two lines of the congruence contained in a general plane of $C^{*}$ and passing through a general point of that plane. Hence the set of lines inside a plane of $C^{*}$ consists of either two pencils of lines or the set of lines tangent to a smooth conic. In the first case we get that the centers of the pencils are in $C$ (otherwise we would get another fundamental curve - which cannot be another line- and we could then repeat the analysis made previously throughout this section). Then the situation would be similar to the case studied just before (and inside) Remark 2.1, but now we would have a correspondence of bidegree $(2, d)$ in the product of $C^{*}$ and $C$, getting a congruence of class $d$. In the second case, we get that the intersection of any plane of $C^{*}$ with the focal locus contains the conic to which the lines of $X$ are tangent, but it can also contain the line $C$ counted with some multiplicity. In other words, if the degree of the focal surface $F$ is $d$, then $F$ has multiplicity $d-2$ at $C$. For instance, taking $F$ to be a general surface for which $C$ has multiplicity $d-2$, the congruence consisting of the lines meeting $C$ and tangent to $F$ (defined by taking the closure of the smooth locus) has order two and class $2 d-2$ (for the class, intersecting with a general plane we get a curve of degree $d$ with a $(d-2)$-fold ordinary point $p$; the class is just the number of lines in that plane passing through $p$ and tangent to the curve at another point).

REMARK 3.2. If a congruence $X$ of order two consists of the lines in a one-dimensional family of pencils, we consider first the set of planes in the pencil. They form an irreducible curve $D \subset \mathbb{P}^{3^{*}}$ which has degree one if the 
general plane contains two different pencils of the congruence, or degree two if the general plane contains only one pencil. In the first case $D$ would consists of a pencil of planes, so that in particular we have a fundamental line, and the situation is as described in Remark 3.1, or more precisely in Proposition 3.2. If instead $D$ has degree two (and hence it is rational), then it consists of the set of planes tangent to a quadric cone $Q \subset \mathbb{P}^{3}$ (with exactly one singular point). On the other hand, the centers of the pencil form a fundamental curve $C \subset \mathbb{P}^{3}$, and we have a map $\varphi: D \rightarrow C$ associating to each plane the center of the (unique) pencil it contains. In particular, $C$ is also rational (as we already knew from the results of this section). A (special) way of constructing congruences of this type is when $C \subset Q$. In particular, take $C$ to be a rational curve of degree $d$ passing with multiplicity $d-2$ through the vertex of $Q$. Then any line of the cone meets $C$ in just one point outside the vertex. We can thus define the congruence consisting of the pencils defined by a tangent plane to $Q$ and the intersection point of $C$ and the tangency line of the plane and $Q$. This congruence has order two and class $d$.

\section{Final remarks}

It is clear that just a slight variation of the above techniques using the focal locus (essentially Lemma 3.1) cannot provide a classification of congruences of some fixed order bigger than two. For instance, in $\mathbb{P}^{3}$ Fano (and Gross with modern techniques) used the so-called triadic locus to study line congruences of order three in $\mathbb{P}^{3}$. It should be mentioned that, while Gross restricted his attention to smooth congruences, Fano (as many other classics) imposed the condition that the congruences do not possess a fundamental curve. And in fact we have seen that the key point to study order two was to prove that for high values of the class there is always a fundamental curve. We thus state the following.

Problem 4.1. Find a function $f$ such that if $b \geq f(a)$ then any line congruence in $\mathbb{P}^{3}$ of order a and class $b$ has a fundamental curve.

The idea (which was accepted as true by the classical geometers) is that the focal surface, which has degree $2 a+2 g_{X}-2$, has also class $2 b+2 g_{X}-2$ (in fact it is possible to compute most of the invariants of the focal surface; see [3]). Hence the class cannot be quite big if $a$ is small and the focal surface is "reasonable". Unfortunately, as shown in [3], there are many ways, 
other than from the fact of having a singular curve corresponding to a fundamental curve, in which the focal surface can have an "unreasonable" behavior (in particular its invariants, including the degree and the class, could be meaningless). We observe that smooth congruences with a fundamental curve has been classified (see [4] for $\mathbb{P}^{3}$, and [1] for a generalization to any $\mathbb{P}^{n}$ ). Thus an answer of Problem 4.1 would produce an answer to the following.

Problem 4.2. Find a function $g$ such that any smooth line congruence in $\mathbb{P}^{3}$ of order $a$ and class $b$ verifies $b \leq g(a)$.

A quadratic bound has been given in [23], while in [13] Gross gives a bound for $b$ of the order of $a^{4 / 3}$. However it is conjectured that, if the congruence is not the set of lines through a point, then $b \leq 3 a$ (which is the best possible linear bound, and it comes from Bogomolov's inequality assuming that the restriction of the universal sub-bundle is semistable). A good reference to learn about the recent developments in this direction is [13] . Notice also that the fact that duality in $\mathbb{P}^{3}$ transforms lines into lines implies that a universal bound $b \leq g(a)$ holds if and only if $a \leq g(b)$ also holds for all smooth congruences.

About line congruences in $\mathbb{P}^{4}$, as we have remarked, even order one is difficult, and in fact it is not clear that even for smooth congruences the class $b$ should be bounded (we have already remarked that the class of smooth congruences of order zero is not bounded).

For order two, it is clear that one could generalize Proposition 3.1, but it is not quite useful, since the fundamental locus could be a surface. And even when restricting to the case of smooth congruences, there is no classification of those with a fundamental surface (in contrast with the case of congruences such that all their lines meet a given curve, which are classified in [1], generalizing the same result for $n=3$ in [4]). It should also be remarked that the important point is not to prove the existence of a fundamental locus, but finding a fundamental locus that is met by all the lines of the congruence. In fact, it is known (see [11]) that smooth line congruences of order two in $\mathbb{P}^{n}$ always have a fundamental locus.

Therefore, for line congruences in $\mathbb{P}^{4}$ (and in general in $\mathbb{P}^{n}$ with $n \geq$ 4), one should not be able to find a bound like in Problem 4.2 (although Problem 4.1 is still valid substituting "fundamental curve" by "curve or surface meeting all the lines of the congruence"). It is then natural to ask which reasonable conditions one can impose to a line congruence in 
order to bound $b$ in terms of $a$. On the other hand, looking at known examples of congruences (see for instance [2]), one finds however that $a$ is almost never bigger than $b+1$. And also most of the congruences for which $a=b+1$ possess a fundamental line meeting all the lines of the congruence. On the other hand, the inequality $a \leq 2 b$ (which would corresponds to Bogomolov's inequality assuming the restriction of the rank-two universal quotient bundle is semistable) also holds, except for the congruence of lines through a point, in all the known examples (and in two of them equality holds). This yields the following problem.

Problem 4.3. Find a function $h$ such that any smooth line congruence in $\mathbb{P}^{4}$ of order $a$ and class $b$ verifies $a \leq h(b)$.

\section{References}

[1] E. Arrondo, M. Bertolini, C. Turrini, Classification of smooth congruences with a fundamental curve, in Projective Geometry and Applications (ed. E. Ballico), Marcel Dekker LN 166 (1994), 43-56.

[2] E. Arrondo, M. Bertolini, C. Turrini, Congruences of small degree in $G(1,4)$, Communications in Algebra 26 (10) (1998), 3249-3266.

[3] E. Arrondo, M. Bertolini, C. Turrini, A focus on focal surfaces, Asian Journal of Mathematics 5 (3) (2001), 535-560.

[4] E. Arrondo, M. Gross, On smooth surfaces in $G r\left(1, \mathbb{P}^{3}\right)$ with a fundamental curve, Manuscripta Math. 79 (1993), 283-298.

[5] C. Ciliberto, E. Sernesi, Singularities of the theta divisor and congruences of planes, Journal of Alg. Geom. 1 no. 2 (1992), 231-250.

[6] P. De Poi, On first order congruences of lines of $\mathbb{P}^{3}$, preprint 1999.

[7] P. DE POI, On first order congruences of lines of $\mathbb{P}^{4}$ with a fundamental curve, Manuscripta Math. 106 (2001), 101-116.

[8] P. De PoI, On first order congruences of lines of $\mathbb{P}^{4}$ with irreducible fundamental surface, preprint 2000.

[9] G. Fano, Sulle congruenze di rette del terzo ordine prive di linea singolare, Att. Acc. di Scienze Torino 29 (1894), 474-493.

[10] G. Fano, Nuove ricerche sulle congruenze di rette del $3^{\circ}$ ordine prive di linea singolare, Memoria della Reale Acc. di Scienze Torino 51 (2) (1902), 1-79.

[11] E. Frigerio, C. Turrini, On order-two line congruences in $\mathbb{P}^{n}$, Arch. Math. 55 (1990), 412-416.

[12] N. Goldstein, The geometry of surfaces in the 4-quadric, Rend. Sem. Mat. Univ. Pol. Torino 43 (3) (1985), 467-499. 
[13] M. Gross, The distribution of bidegrees of smooth surfaces in $G\left(1, \mathbb{P}^{3}\right)$, Math. Ann. 292 (1992), 127-147.

[14] M. GRoss, Surfaces of bidegree $(3, n)$ in $\operatorname{Gr}\left(1, \mathbb{P}^{3}\right)$, Math. Z. 212 (1993), 73106.

[15] E.E. Kummer, Über die algebraischen Strahlensysteme insbesorende über die ersten und zweiten Ordnung, Abh. Akad. Wiss. Berlin, 1866.

[16] H. MAEDA, Nef line bundles on algebraic surfaces, Kodai Math. J. 18 (1995), 187-197.

[17] G. Marletta, Sui complessi di rette del primo ordine dello spazio a quattro dimensioni, Rend. Circ. Matematico Palermo 28 (1909), 353-399.

[18] G. Marletta, Sopra i complessi di rette d'ordine uno dell'S $S_{4}$, Atti Accad. Gioenia di Catania 5 vol. III (1910).

[19] M. Pedreira, L. Solá-Conde, Projective generation and smoothness of congruences of orden one, preprint 2000.

[20] Z. RAN, Surfaces of order 1 in grassmannians, Crelle J. reine angew. Math. 368 (1986), 119-126.

[21] E. RogorA, Varieties with many lines, Manuscripta Math. 82 (1987), 207220.

[22] B. Segre, Sulle $V_{n}$ contenenti più $d i \infty^{n-k} S_{k}$, Lincei - Rend. Sc. Fis. Mat. e Nat. 5 (1948), 193-197 and 275-280.

[23] A. Verra, Geometria della retta in dimensione 2, unpublished paper (1986).

[24] F.L. Zak, A.V. Inshakov, S.M. L'vovski, A.A. Oblomkov, On congruences of lines of order one in $\mathbb{P}^{3}$, preprint 2000.

Enrique Arrondo

Departamento de Álgebra

Facultad de Ciencias Matemáticas

Universidad Complutense de Madrid

E-28040 Madrid, Spain 